\section{Loss of recent memory after bilateral hippocampal lesions: memory and memories-looking back and looking forward}

\author{
Brenda Milner, Denise Klein
}

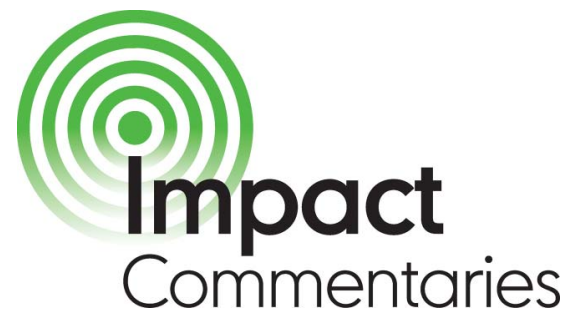

In 1957, Scoville and Milner reported a series of case studies describing the nature of the memory defect they observed following bilateral surgical removals from the medial temporal region. The paper highlighted that extensive bilateral surgical excisions from the hippocampus and hippocampal gyrus resulted in severe and lasting memory loss; it also focused attention on these brain structures as critical for the maintenance of normal memory function.

These findings ran counter to prevailing views at the time by linking a memory disturbance to damage to a specific part of the brain, and highlighted the fact that the hippocampal region was essential in the establishment of new and enduring memories. Milner had been invited to study this series of patients by the neurosurgeon, William Scoville, following a report by Milner and Penfield ${ }^{1}$ of two patients who showed unexpected memory loss following unilateral left-temporal lobe brain operations performed at the Montreal Neurological Hospital. To explain this memory loss, Milner and Penfield ${ }^{1}$ had speculated that in each case there had been a pre-existing unsuspected lesion in the hippocampal region of the opposite hemisphere, so that the operation effectively deprived the patients of hippocampal function bilaterally. This was confirmed 12 years later in the case of $\mathrm{PB}$, when

Montreal Neurological Institute, Neurology and Neurosurgery, McGill University, Montreal, Quebec, Canada

Correspondence to Dr Brenda Milner, Montreal Neurological Institute, 3801 University Street, Montreal, Quebec, Canada, H3A 2B4; brenda.milner@mcgill.ca
Loss of recent memory after bilateral hippocampal lesions

Authors: Scoville WB, Milner B

Year published: 1957

Total cites: 5284

autopsy findings revealed that he had had longstanding right hippocampal atrophy. The hippocampal region was determined to be important, because PB had had his temporal lobectomy in two stages, 5 years apart, and Milner and Penfield noted that it was only after the medial removal that the memory loss occurred.

The paper by Scoville and Milner ${ }^{2}$ complemented that of Milner and Penfield, in that the damage in each case was known to be bilateral; but its real impact was that they were able to demonstrate that gradations of memory loss related to the estimated extent of surgical excision. At a time without the aid of brain imaging, Scoville carefully delineated the extent of removal, and Milner determined the relationship between extent of removal and the kind and severity of memory loss observed. Having the same surgeon across a range of cases allowed for consistency within the frame of reference, thus permitting inferences to be drawn about the link between a welldefined focal lesion and specific memory impairments. It was clear that patients with hippocampal lesions retained their professional knowledge and skills, their understanding and use of language, and their ability to recall early experiences. What these patients had was continuous anterograde amnesia, forgetting of events as they lived them, of life as they lived it. The data emphasised that the hippocampus and hippocampal gyrus play an essential part in the consolidation process by means of which daily experiences are transformed into enduring memories. In particular, the now famous case of Henry Molaison (then known only as HM) was critical in defining the role of the hippocampal zone in the learning process; but it also called for caution in the clinical setting, highlighting the necessity to look carefully for possible signs of bilateral temporal- lobe abnormality in all patients being considered for a unilateral temporal lobectomy.

Reflecting back, the paper takes an important place in history by demonstrating a clear relationship between the location and extent of the lesion and the memory loss in young individuals with no previous memory impairment. This early emphasis on the role of the medial temporal lobes in memory remains central to all interpretations of the amnestic syndrome and highlights the important role that neuropsychological testing plays in providing a window into the brain. This was the case in 1957, long before the advent of brain-imaging tools, and is still the case today.

Looking forward, it is now possible to use functional neuroimaging techniques to observe the normal brain in action during the performance of cognitive tasks and to use structural brain imaging to explore the contribution of different medial temporallobe structures to memory processes. Before brain imaging, clearly we had less information, but brain imaging tools are complex and sifting through the data can yield too much, making it difficult to disambiguate the role of different brain regions. The challenge now is to link the lesion work and imaging to understand the brain in health and in disease. The task for the future is to understand the brain connections that help us to remember our past and make the memories that we bring to our future.

Competing interests None declared.

Provenance and peer review Commissioned; internally peer reviewed.

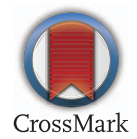

To cite Milner B, Klein D. J Neurol Neurosurg Psychiatry 2016;87:230.

Received 2 July 2015

Accepted 22 July 2015

Published Online First 17 August 2015

J Neurol Neurosurg Psychiatry 2016;87:230. doi:10.1136/jnnp-2015-311092

\section{REFERENCES}

1 Milner B, Penfield W. The effect of hippocampal lesions on recent memory. Trans Am Neurol Assoc 1955;80:42-8.

2 Scoville WB, Milner B. Loss of recent memory after bilateral hippocampal lesions. I Neurol Neurosurg Psychiatry 1957;20:11-21. 